\title{
Small-mass effects in heavy-to-light form factors
}

\author{
T. Engel, ${ }^{a}$ C. Gnendiger, ${ }^{a}$ A. Signer ${ }^{a, b}$ and Y. Ulrich ${ }^{a, b}$ \\ ${ }^{a}$ Paul Scherrer Institut, \\ CH-5232 Villigen PSI, Switzerland \\ ${ }^{b}$ Physik-Institut, Universität Zürich, \\ Winterthurerstrasse 190, CH-8057 Zürich, Switzerland \\ E-mail: tim.engel@psi.ch, christoph.gnendiger@psi.ch, \\ adrian.signer@psi.ch, yannick.ulrich@psi.ch
}

ABSTRACT: We present the heavy-to-light form factors with two different non-vanishing masses at next-to-next-to-leading order and study its expansion in the small mass. The leading term of this small-mass expansion leads to a factorized expression for the form factor. The presence of a second mass results in a new feature, in that the soft contribution develops a factorization anomaly. This cancels with the corresponding anomaly in the collinear contribution. With the generalized factorization presented here, it is possible to obtain the leading small-mass terms for processes with large masses, such as muon-electron scattering, from the corresponding massless amplitude and the soft contribution.

KEYwords: Effective Field Theories, Perturbative QCD, Renormalization Regularization and Renormalons, Scattering Amplitudes

ArXiv ePrint: 1811.06461 


\section{Contents}

1 Introduction 1

2 Notation and setup 3

2.1 Expansion in the small mass 4

2.2 Dimensional schemes and $n_{\epsilon} \mathrm{QCD} \quad 4$

3 Results 5

3.1 Result with the full mass dependence and IR prediction 6

3.2 Expanded result 7

$\begin{array}{lll}3.3 & \text { Factorization } & 7\end{array}$

4 Comparison with heavy-quark form factor $\quad 11$

5 Conclusions 13

$\begin{array}{ll}\text { A Master integrals } & 14\end{array}$

\section{Introduction}

Perturbative calculations in QED and QCD are often carried out setting fermion masses to zero. This reduces the number of scales and results in a simplification of the computation of the virtual corrections. However, the neglect of fermion masses also has a profound impact on the structure of the infrared (IR) singularities. In the case of QED, fermion masses $m \neq$ 0 can be considered as a regulator for collinear singularities and only soft (and ultraviolet) singularities remain. Collinear singularities, that in dimensional regularization are manifest as poles $(1 / \epsilon)^{n}$, are then replaced by terms $\log \left(\mathrm{m}^{2} / \mathrm{s}\right)$, where $s$ is a kinematic invariant. Due to the non-Abelian nature of gluon interactions the situation is more involved in QCD. However, the structure of IR singularities in massless theories [1, 2] is altered through quark masses [3] in a similar way.

It is not possible to obtain a complete amplitude with $m \neq 0$ from the corresponding amplitude with $m=0$ without performing a full computation. The universal structure of IR singularities, however, enables the extraction of the leading $\log \left(\mathrm{m}^{2} / \mathrm{s}\right)$ terms from the massless amplitudes. If the mass is small compared to all other kinematic invariants, this can provide a reasonable approximation. In fact, such relations have been worked out already at next-to-next-to-leading order (NNLO). Initially this was done for QED in the context of Bhabha scattering [4]. Later, a more general approach has been presented [5, 6] that relies on factorization and is also valid in QCD. Very recently, these considerations have been extended beyond NNLO, in particular for the heavy-quark form factor [7-9]. 
In this article we extend the previous NNLO analyses to the case of two different external masses with $M \gg m$. We provide an IR factorization formula that allows to obtain the logarithmic corrections $\log \left(m^{2} / M^{2}\right)$ and $\log \left(m^{2} / s\right)$ from the amplitudes with $m=0$. As it turns out, diagrams with fermion loops play a special role and lead to complications that are absent if there is no second (large) mass.

This extension is particularly relevant in light of the recently proposed experiment MUONE at CERN to measure muon-electron scattering at high precision. Precise data of the angular distribution of this process will provide an alternative approach to measure the hadronic vacuum polarization $[10,11]$. In order to achieve this, the theoretical predictions have to be known with sufficient accuracy. While a fully differential NLO calculation is available [12] and the contribution of the hadronic vacuum polarization has been evaluated at NNLO [13, 14], a full NNLO computation is still missing. So far, the two-loop integrals of $\mu-e$ scattering have been calculated with vanishing electron mass $[15,16]$. This enables the computation of the two-loop amplitude for massless electrons. However, the necessary theoretical accuracy of $10^{-5}$ requires the inclusion of the leading electron mass effects.

As a concrete example, we consider the effects of a small final-state mass to the heavyto-light form factor, i. e. the transition between a heavy fermion of mass $M$ to a lighter one of mass $m$. While one motivation is to obtain the generalized factorization formula mentioned above, the heavy-to-light form factor is an interesting quantity in itself. It is an important ingredient in several precise probes of the Standard Model. In the context of the muon decay, it is one of the most precisely measured quantities in particle physics and is used to extract for example the Fermi constant $G_{F}$. But also $b$ and top quark decays play an important role in precision tests of the Standard Model.

Therefore, the QED and QCD corrections to the heavy-to-light form factor are needed at high precision. At the one-loop level, the QED correction to the muon decay with full final-state mass dependence has been known for many decades [17, 18]. The muon decay branching ratio was then calculated at two-loops in QED using the optical theorem [19]. The electron energy spectrum including leading electron mass effects was obtained a few years later $[20,21]$. In 2008 the full electron mass dependence of the energy spectrum was computed numerically at NNLO in QED [22]. For vanishing final-state masses, analytic expressions for two-loop QCD corrections to the heavy-to-light form factors have been calculated [23-26] in several regularization schemes [27].

Recently, the master integrals needed to compute the form factors for arbitrary masses have been presented [28] in terms of generalized polylogarithms (GPL) [29]. Using these integrals, we calculate the NNLO form factor for two different non-vanishing fermion masses. However, the numerical evaluation of the GPLs is time consuming and, therefore, difficult to implement in a full NNLO Monte Carlo. Fortunately, the physical mass ratios $m_{e} / m_{\mu}$, $m_{b} / m_{t}$, and $m_{s} / m_{b}$ are small enough to allow for an expansion in the small fermion mass. We perform such an expansion, write it in a factorized form and compare it to the result with the full mass dependence. All calculations are conducted in a generic way that allows to extract the results in various dimensional regularization schemes.

This paper is structured as follows: in section 2 we introduce our notation and describe our setup, including a review of the regularization schemes used. Then, in section 3 we 
discuss our results for the mass effects in the heavy-to-light form factors and present the factorization. Lengthy results are provided in the supplementary material. In section 4 we compare our results to previous factorization formulations and discuss the extension of our approach to other processes like the heavy-quark form factor and $\mu-e$ scattering. Finally, we conclude in section 5 .

\section{$2 \quad$ Notation and setup}

The decay of a fermion with momentum $p^{2}=M^{2}$ into a lighter fermion with momentum $q^{2}=m^{2}$ is closely related to the amplitude $\mathcal{A}^{\rho}=\bar{u}(q) \Gamma^{\rho} u(p)$. The vertex can be written in terms of form factors $F_{i}$ and $G_{i}$ as

$$
\begin{aligned}
\Gamma^{\rho}= & F_{1} \gamma^{\rho}+\frac{i}{2 M} \sigma^{\rho \sigma} Q_{\sigma} F_{2}+\frac{1}{2 M} Q^{\rho} F_{3} \\
& +G_{1} \gamma_{5} \gamma^{\rho}+\frac{i}{2 M} \gamma_{5} \sigma^{\rho \sigma} Q_{\sigma} G_{2}+\frac{1}{2 M} \gamma_{5} Q^{\rho} G_{3},
\end{aligned}
$$

with $Q=p-q$. There are different conventions used in the literature for the form factors $G_{i}$. In (2.1) they are defined such that each $G_{i}$ is closely related to the corresponding $F_{i}$. Exploiting the symmetry of the Lagrangian under the simultaneous transformation of the light fermion field $\psi_{q} \rightarrow \gamma_{5} \psi_{q}$ and $m \rightarrow-m$, the axial form factors can be obtained from the vector form factors through [30]

$$
G_{i}(m)=F_{i}(-m)
$$

Therefore, we restrict ourselves to the discussion of the $F_{i}$. Furthermore, since it has the most interesting IR structure, we focus on $F_{1}$ and comment on $F_{2}$ and $F_{3}$ occasionally.

We parametrize the result in terms of the three kinematic invariants of the process

$$
M, \quad z=\frac{m}{M}, \quad \text { and } \quad x=\frac{Q^{2}}{M^{2}} .
$$

For convenience we also define

$$
s=2 p \cdot q=M^{2}(1-x)+m^{2}
$$

and write the dependence on the kinematics of the form factors as $F_{i}(M, m, s)$.

The ultraviolet (UV) renormalization of the masses and wave functions of the external particles is performed in the on-shell scheme, while the QED and QCD couplings are renormalized in the $\overline{\mathrm{MS}}$ scheme. The renormalization constants from $[27,31,32]$ are extended to account for $n_{f}$ massless flavours, $n_{m}$ flavours with mass $m$, and $n_{h}$ flavours with mass $M$.

The form factors $F_{i}$ in (2.1) receive contributions from one one-loop and 9 (13) twoloop diagrams in QED (QCD), including fermion loops with masses $M$ and $m$ as well as massless ones. Writing all tensor integrals in terms of scalar ones, this results in about 700 scalar loop integrals. Using the program reduze [33] which implements Laporta's algorithm [34], these reduce to a set of 40 two-loop and three one-loop master integrals. 


\subsection{Expansion in the small mass}

The form factors can be written as a function of the three invariants $m, M$, and $s$. To obtain the full dependence on all invariants, we use the integrals from [28]. However, as mentioned above, the numerical evaluation of the GPLs $[35,36]$ is rather time consuming. For physical applications we therefore consider an expansion in

$$
m \ll M \sim s \sim Q^{2},
$$

which corresponds to an expansion in $z$. Obviously, the algebraic part of the expression can be expanded trivially. In order to obtain the expanded form of the master integrals, we use the method of regions [37].

Introducing two light-like directions $e_{\mu}=\left(1,0_{\perp},+1\right) / \sqrt{2}$ and $\bar{e}_{\mu}=\left(1,0_{\perp},-1\right) / \sqrt{2}$ with a $(d-2)$-dimensional perpendicular component, ${ }^{1}$ we parametrize the external momenta as

$$
\begin{aligned}
q^{\mu} & =\sqrt{2} E e^{\mu}+q_{\perp}^{\mu}, \\
p^{\mu} & =\frac{M}{\sqrt{2}}\left(e^{\mu}+\bar{e}^{\mu}\right),
\end{aligned}
$$

with $\left(q_{\perp}\right)^{2}=m^{2}$ and $s=2 M E$. To obtain the expansion, we attach a small scaling parameter $\lambda \ll 1$ to $m$ and $q_{\perp}$. In light-cone coordinates $k=\left(k_{+}, k_{-}, k_{\perp}\right)$ with $k_{+}=(k \cdot e)$ and $k_{-}=(k \cdot \bar{e})$ the external momenta then scale as $q \sim(0,1, \lambda)$ and $p \sim(1,1,0)$. The momentum regions that contribute to the integrals are associated with the following scalings of the integration momenta:

$$
\begin{aligned}
\text { hard: } & k \sim(1,1,1) \\
\text { soft: } & k \sim(\lambda, \lambda, \lambda) \\
\text { collinear: } & k \sim\left(\lambda^{2}, 1, \lambda\right) \\
\text { ultrasoft: } & k \sim\left(\lambda^{2}, \lambda^{2}, \lambda^{2}\right)
\end{aligned}
$$

Expanding all master integrals in these regions, the form factors can be written as a series in $\lambda$ (i. e. $z$ ), in principle to any order. Details of the calculation of the master integrals in expanded form are given in appendix A. As it turns in section 3.3, restricting this expansion to leading order in $\lambda$, counting $\log \lambda \sim 1$, the form factors actually factorize and can be written as a product of hard, soft, and collinear contributions. All ultrasoft contributions cancel between diagrams.

\subsection{Dimensional schemes and $n_{\epsilon} \mathrm{QCD}$}

In order to regularize UV and IR divergent integrals, we use dimensional regularization (DREG) $[38,39]$ throughout the paper. More precisely, we formally consider space-time and momentum integrations in an arbitrary dimension

$$
d=4-2 \epsilon
$$

\footnotetext{
${ }^{1}$ Note that this definition differs from the usual light-cone vector by a factor of $\sqrt{2}$.
} 
and indicate this shift by a subscript, for example $\partial_{[4]}^{\mu} \rightarrow \partial_{[d]}^{\mu}$ and $k_{[4]}^{\mu} \rightarrow k_{[d]}^{\mu}$ for derivatives and loop momenta, respectively. Depending on the specific realization of DREG, the (quasi) dimensionality of other algebraic objects like metric tensors, $\gamma$ matrices, and vector fields might or might not be different from $d$. To account for this regularization-scheme dependence, we write gauge fields associated to particles in 1PI diagrams as

$$
A_{[4]}^{\mu, a} \rightarrow A_{[d]}^{\mu, a}+A_{\left[n_{\epsilon}\right]}^{\mu, a},
$$

where $A_{[d]}^{\mu, a}$ is a (quasi) $d$-dimensional gauge field and $A_{\left[n_{\epsilon}\right]}^{\mu, a}$ is a so-called $\epsilon$-scalar field. Considering $n_{\epsilon}$ as an initially arbitrary quantity, the regularization-scheme dependence is manifest in terms $\propto n_{\epsilon}$. In this way it is possible to consider the most commonly used dimensional schemes simultaneously, i. e. the 't Hooft-Veltman scheme (HV) [39], conventional dimensional regularization (CDR) [40], the four-dimensional helicity scheme (FDH) [41], and dimensional reduction (DRED) [42]. These schemes are defined such that the value of $n_{\epsilon}$ is given by

$$
\begin{aligned}
\text { HV, CDR : } & n_{\epsilon}=0 \\
\text { FDH, DRED : } & n_{\epsilon}=2 \epsilon .
\end{aligned}
$$

According to the split in (2.9), the QCD covariant derivative can be written as

$$
D_{[4]}^{\mu} \psi_{i} \rightarrow \partial_{[d]}^{\mu} \psi_{i}+i\left(g_{s}^{0} A_{[d]}^{\mu, a}+g_{e}^{0} A_{\left[n_{\epsilon}\right]}^{\mu, a}\right) T_{i j}^{a} \psi_{j}
$$

where $g_{e}^{0}$ is the bare coupling of $\epsilon$-scalars to fermions. This so-called evanescent coupling is only present in FDH and DRED and has to be introduced at the Lagrangian level as it is not protected by Lorentz and gauge invariance. Its UV renormalization is therefore different compared to the one of the gauge coupling $g_{s}^{0}$ [43]. Only after UV renormalization, the numerical values of the renormalized couplings can be set equal. In the following both couplings are used in the form $\alpha_{i}^{0}=\left(g_{i}^{0}\right)^{2} /(4 \pi)$ with $i \in\{s, e\}$. The corresponding renormalized couplings are denoted by $\alpha_{i}$.

In what follows we keep the dependence on $\alpha_{s}, \alpha_{e}$, and $n_{\epsilon}$ in the results and refer to this as $n_{\epsilon} \mathrm{QCD}$. This allows one to obtain the corresponding results in all schemes through (2.10). For a comprehensive definition and a review of the different dimensional schemes we refer to [44] and references therein.

\section{Results}

In this section we present the result of the form factor $F_{1}(M, m, s)$. We begin by keeping the full mass dependence of $F_{1}$ and check its IR structure to verify our calculation. We then discuss the expanded result, which, due to the large scale separation between $M$ and $m$, is sufficient for most practical purposes. In fact, we show that the leading term of this expansion represents an excellent approximation and leads to a factorized form. 


\subsection{Result with the full mass dependence and IR prediction}

We calculated the form factor $F_{1}(M, m, s)$ at NNLO with full mass dependence using [28]. The result can be expressed in terms of two dimensionless variables but involves a huge number of terms. ${ }^{2}$ However, the master integrals take a particularly simple form if one of the variables is chosen to be $\chi$, defined as

$$
\chi+\frac{1}{\chi}=\frac{2 p \cdot q}{M m}=\frac{s}{M m}=\frac{1-x}{z}+z .
$$

This agrees with $\chi$ as defined in [28]. The usefulness of $\chi$ can be understood by noting that the singularities of $F_{1}(M, m, s)$ can be expressed in terms of UV singularities of Wilson lines [3] with scaling symmetries $p \rightarrow \lambda_{1} p, q \rightarrow \lambda_{2} q$. Indeed, $\chi$ is invariant under these symmetries, whereas $s$ is only invariant under the restricted case $\lambda_{1} \lambda_{2}=1$. More precisely, the poles of $F_{1}(M, m, s)$ are governed by the velocity-dependent cusp anomalous dimension, which is a function of the cusp angle $\beta_{p q}=\log (\chi)$. Therefore, the poles of $F_{1}(M, m, s)$ depend only on $\chi$. The finite part, however, also depends on the second variable $z$.

The result can now be expressed using 540 different GPLs of the form $G\left(\left\{\alpha_{i}\right\}, 1\right)$, $G\left(\left\{\alpha_{i}\right\}, \chi\right)$ and $G\left(\left\{\alpha_{i}\right\}, z\right)$. A set of eleven letters $\alpha_{i}$ are needed to express the finite part of $F_{1}(M, m, s)$, namely

$$
\alpha_{i} \in\left\{0, \pm 1, \pm z, \pm \frac{1}{z}, \pm\left(z-\sqrt{1-z^{2}}\right), \frac{1 \pm \sqrt{1-z^{2}}}{z}\right\}
$$

The divergent part contains only simple polylogarithms of $\chi$, i.e. the alphabet is $\{0, \pm 1\}$. In fact, the divergent part can be checked by considering the IR anomalous dimensions presented in $[27,31]$. To do this, we construct $\log (\mathbf{Z})$ as

$$
\left.\log F_{1}\right|_{\text {poles }}=\left\{F_{1}^{(1)}+\left[F_{1}^{(2)}-\frac{1}{2}\left(F_{1}^{(1)}\right)^{2}\right]\right\}_{\text {poles }}+\mathcal{O}\left(\alpha_{i}^{3}\right) \equiv \log (\mathbf{Z}),
$$

where $F_{1}^{(l)}$ is the $l$-loop contribution to $F_{1}$. Using the explicit expressions for the twoloop heavy-quark anomalous dimension $\gamma_{Q}$ and the velocity-dependent cusp anomalous dimension $\gamma_{\text {cusp }}(\log \chi)$ in FDH, as well as the one-loop $\beta$-functions of $n_{\epsilon} \mathrm{QCD}$ (see [27] and references therein), we can predict all poles of $\log (\mathbf{Z})$. The leading poles expressed in terms of the renormalized couplings of $n_{\epsilon} \mathrm{QCD}$ read

$$
\begin{aligned}
\log (\mathbf{Z})= & \left(\frac{\alpha_{s}}{4 \pi}\right) C_{F} \frac{1}{2 \epsilon}\left[\frac{\chi^{2}+1}{\chi^{2}-1} \log (\chi)-1\right] \\
& +\left(\frac{\alpha_{s}}{4 \pi}\right)^{2}\left[\frac{1}{\epsilon^{2}} \frac{C_{F} C_{A}\left(n_{\epsilon}-22\right)+4 C_{F} n_{f}}{24}\left(\frac{\chi^{2}+1}{\chi^{2}-1} \log (\chi)-1\right)+\mathcal{O}\left(\epsilon^{-1}\right)\right] .
\end{aligned}
$$

The result of our explicit computation agrees with this prediction. Since the full NNLO result of $\log (\mathbf{Z})$ is too complicated to be listed here it is presented in the supplementary material. The result is given in $n_{\epsilon} \mathrm{QCD}$, but there are no terms involving $\alpha_{e}$ and no scheme dependence at the one-loop level.

\footnotetext{
${ }^{2}$ The expression is approximately $1 \mathrm{~GB}$.
} 
Beyond the first order in QED with $n_{f}=0$, the expression for $\log F_{1}(M, m, s)$ is finite since all the divergences of the form factors are described by exponentiating the one-loop soft poles. Therefore, after taking the limit $\epsilon \rightarrow 0$, there is no scheme dependence in $\log F_{1}(M, m, s)$.

\subsection{Expanded result}

Once the result is expanded in $z=m / M$, it becomes significantly simpler. The alphabet (3.2) is now only $\{0, \pm 1\}$, i.e. the result can be expressed completely in terms of the harmonic polylogarithms [45], which are implemented in the Mathematica package HPL [46]. However, some master integrals can be written in a more compact form by keeping some specific GPLs with weight two [23].

The poles can now be written as

$$
\begin{aligned}
& \log (\mathbf{Z})=\left(\frac{\alpha_{s}}{4 \pi}\right) \frac{C_{F}}{2 \epsilon} L \\
&+\left(\frac{\alpha_{s}}{4 \pi}\right)^{2}\left(C_{A} C_{F}\left\{\frac{n_{\epsilon}-22}{24 \epsilon^{2}} L+\frac{1}{\epsilon}\left[\left(\frac{67}{36}-\frac{n_{\epsilon}}{9}-\frac{1}{2} \zeta_{2}\right) L+\frac{1}{2}-\frac{1}{2} \zeta_{3}\right]\right\}\right. \\
&+\left.C_{F} n_{F}\left\{\frac{1}{6 \epsilon^{2}} L-\frac{5}{18 \epsilon} L\right\}\right)+\mathcal{O}\left(\alpha_{i}^{3}, z^{2}\right),
\end{aligned}
$$

with

$$
L=\log \left(\frac{s}{m M}\right)-1 .
$$

The complete expression for $F_{1}(M, m, s)$ as well as $F_{2}(M, m, s)$ and $F_{3}(M, m, s)$ is provided in the supplementary material attached to the paper.

Using the method of regions, it is not only possible to calculate the leading terms of $\mathcal{O}\left(z^{0}\right)$ but also subleading terms. In particular, the term of $\mathcal{O}\left(z^{1}\right)$ can be calculated easily because, apart from trivial terms from the Dirac algebra, only soft integrals can generate terms odd in $z$. We note that, because of the symmetry (2.2), terms of $\mathcal{O}\left(z^{1}\right)$ drop out in the full matrix element for decay processes.

We can assess the reliability of our expansion by comparing the full result of $\left.\log F_{1}\right|_{\alpha_{i}^{2}}$ to its expansion in QED. We do this in figure 1 numerically, taking into account terms of $\mathcal{O}\left(z^{0}\right)$ and subsequently of $\mathcal{O}\left(z^{1}\right)$. The GPLs have been evaluated using an in-house implementation based on $[35,36]$. At the physical mass ratio $z=m_{e} / m_{\mu}$, that is particularly relevant for MUONE, the relative error is approximately $10^{-3}$ at $\mathcal{O}\left(z^{0}\right)$ and $<10^{-4}$ at $\mathcal{O}\left(z^{1}\right)$. In figure 2 we present the kinematic dependence of $F_{1}(M, m, s)$. Note that around $x=0.4$ the difference between the full mass dependence and the expansion up to $\mathcal{O}\left(z^{1}\right)$ crosses zero. For values of $x \rightarrow 1$, the agreement gets worse since the expansion in $\mathrm{m}^{2} / \mathrm{s}$ breaks down, as can be seen from (2.4).

\subsection{Factorization}

Motivated by soft-collinear effective theory (SCET) [47-49], it is expected that at leading order in $z=m / M$ the massive form factor $F_{1}(M, m, s)$ factorizes into hard, soft, and 


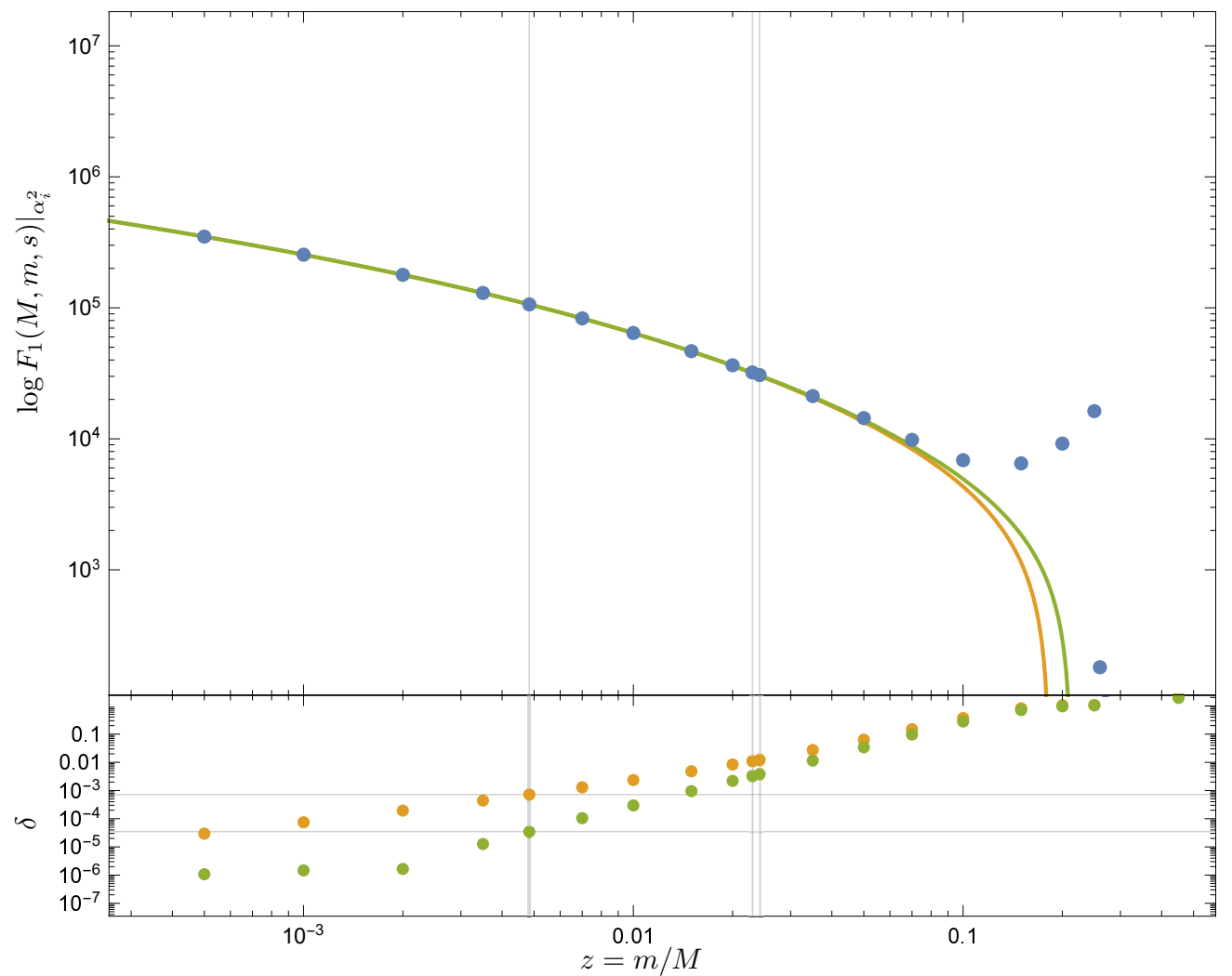

Figure 1. Comparison of $\left.\log \left(F_{1}\right)\right|_{\alpha_{i}^{2}}$ as a function of $z$ in QED at two loops using the exact integrals [28] (blue) as well as the expansion at $\mathcal{O}\left(z^{0}\right)$ (orange) and $\mathcal{O}\left(z^{1}\right)$ (green) in the upper panel. The lower panel shows the relative difference. The vertical lines indicate the physical mass ratios $m_{e} / m_{\mu}, m_{s} / m_{b}$ and $m_{b} / m_{t}$.

collinear parts according to

$$
F_{1}(M, m, s)=\sqrt{Z_{q}} \times \mathcal{S} \times F_{1}(M, 0, s)+\mathcal{O}(m / M)
$$

The hard part corresponds to $F_{1}(M, 0, s)$ since using the scaling (2.7a) simply results in neglecting $m$ in the integrand.

For the soft part $\mathcal{S}$, we only need to consider diagrams with internal fermions. Indeed, by performing the formal decoupling of gluon and fermion fields in the SCET framework, one can show that purely gluonic contributions to the soft part vanish to all orders [6, 47]. A simple counting argument implies that only the fermion bubble with mass $m$ contributes (cf. figure 3). Therefore, the unrenormalized soft part $\mathcal{S}^{0}$ can easily be calculated from first principle in the SCET framework using (2.7b), i.e.

$$
\mathcal{S}^{0}=1+\left(\frac{\alpha_{s}^{2}}{4 \pi}\right) C_{F} \int[\mathrm{d} k] \frac{\left(-2 p^{\mu}\right)\left(-2 q_{-}^{\nu}\right)}{\left(k^{2}\right)^{2}(2 p \cdot k)\left(2 q_{-} \cdot k\right)} \Pi_{\mu \nu}^{\left(n_{m}\right)}(k),
$$

where the integral measure is defined in (A.1). In accordance with (2.7b), we only use the large component of the collinear momentum $q$. Because $\epsilon$-scalars do not couple to fermions 


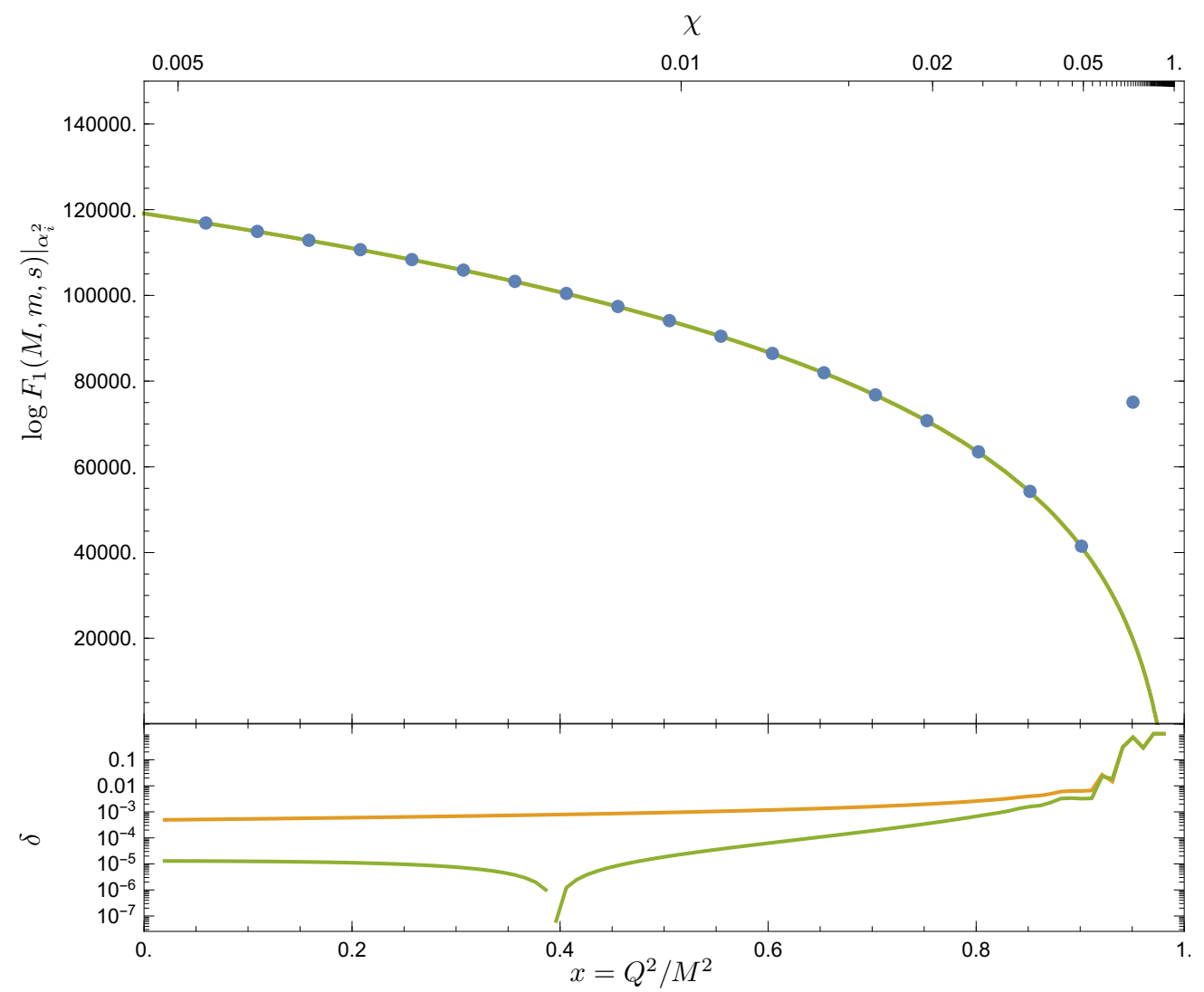

Figure 2. The kinematic dependence of $\left.\log \left(F_{1}\right)\right|_{\alpha_{i}^{2}}$ in QED with the full mass dependence (blue) as well as the expansion at $\mathcal{O}\left(z^{0}\right)$ (orange) and $\mathcal{O}\left(z^{1}\right)$ (green). The lower panel shows the relative difference. Note that around $x=0.4$ the deviation between the exact result and the $\mathcal{O}\left(z^{1}\right)$ result crosses zero.

in the eikonal approximation [27], there is no contribution $\propto n_{\epsilon}$. The function $\Pi_{\mu \nu}^{\left(n_{m}\right)}$ is the contribution of $n_{m}$ fermions with mass $m$ to the usual tensorial vacuum polarization. When calculating $\mathcal{S}^{0}$, one encounters an anomaly, i. e. the breaking of naive factorization [50, 51]. Following [52], we call this factorization anomaly. ${ }^{3}$ This is a new feature that is only present due to the large mass $p^{2}=M^{2}$. As we discuss in section 4 , if the heavy fermion is replaced by a fermion with mass $m$ or a massless one, the soft contributions vanish and, therefore, have no factorization anomaly.

The factorization anomaly can be regularized by shifting the power of the propagator $p \cdot k$ at the diagrammatic level according to $[54,55]$

$$
\frac{1}{(k-p)^{2}-M^{2}+\mathrm{i} 0^{+}} \rightarrow \frac{1}{-2 p \cdot k+\mathrm{i} 0^{+}} \rightarrow\left(-\nu^{2}\right)^{\eta} \frac{1}{\left(-2 p \cdot k+\mathrm{i} 0^{+}\right)^{1+\eta}},
$$

where the regulator $\eta$ has to be expanded before the dimensional regulator $\epsilon$. This regularization also introduces an associated scale $\nu$ that drops out in the final result. The

\footnotetext{
${ }^{3}$ This is also referred to as collinear anomaly or rapidity divergence [53].
} 


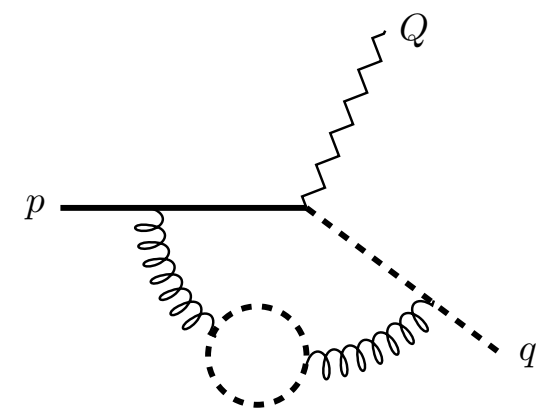

Figure 3. The $n_{m}$ bubble giving rise to the soft contribution. Solid lines are fermions with mass $M$ and dashed lines are fermions with mass $m$.

only further soft contribution is from $n_{m}$ terms in the wave-function renormalization of the heavy fermion. Including this contribution, $Z_{2}^{\mathcal{S}}$, we obtain

$$
\begin{aligned}
\mathcal{S}=\sqrt{Z_{2}^{\mathcal{S}}} \times \mathcal{S}^{0}=1+\left(a_{s}^{0}(M m)\right)^{2} C_{F} n_{m}[ & \frac{1}{\eta}\left(-\frac{2}{3 \epsilon^{2}}+\frac{10}{9 \epsilon}-\frac{56}{27}-\frac{4}{3} \zeta_{2}\right) \\
& +\frac{1}{2 \epsilon^{3}}-\frac{1}{9 \epsilon^{2}}+\frac{1}{\epsilon}\left(-\frac{26}{27}+\zeta_{2}\right) \\
& \left.+\frac{11}{3}-\frac{2}{3} \zeta_{3}-\frac{2}{9} \zeta_{2}\right]+\mathcal{O}\left(a_{s}^{3}, \epsilon, \eta\right),
\end{aligned}
$$

where we define $a_{s}^{0}(x)$ as

$$
a_{i}^{0}(x)=\left(\frac{\alpha_{i}^{0}}{4 \pi}\right)\left(\frac{\mu^{2}}{m^{2}}\right)^{\epsilon}\left(-2+\mathrm{i} 0^{+}\right)^{\eta / 2}\left(\frac{-\nu^{2}}{x}\right)^{\eta / 2}, \quad i \in\{s, e\},
$$

with an analogous expression for the renormalized couplings.

The calculation of the collinear terms, $Z_{q}$, is more involved because the integral reduction mixes different momentum regions and is of limited use in the presence of the regulator $\eta$. Therefore, we have explicitly calculated the collinear gluon contribution to the process by expanding the diagrams using $(2.7 \mathrm{c})$ without any integral reduction. This way, $Z_{q}$ can be written in terms of the bare couplings of $n_{\epsilon} \mathrm{QCD}$ as

$$
\begin{gathered}
\sqrt{Z_{q}=}+a_{s}^{0} C_{F}\left\{\frac{1}{\epsilon^{2}}+\frac{1}{2 \epsilon}+\zeta_{2}+2+\left(4+\frac{1}{2} \zeta_{2}\right) \epsilon+\left(8+2 \zeta_{2}+\frac{7}{4} \zeta_{4}\right) \epsilon^{2}+\mathcal{O}\left(\epsilon^{3}\right)\right\} \\
+a_{e}^{0} C_{F} \frac{n_{\epsilon}}{4}\left\{-\frac{1}{\epsilon}-1-\left(1+\zeta_{2}\right) \epsilon-\left(1+\zeta_{2}\right) \epsilon^{2}+\mathcal{O}\left(\epsilon^{3}\right)\right\} \\
+\left(a_{s}^{0}(s)\right)^{2}\left\{C _ { F } ^ { 2 } \left[\frac{1}{2 \epsilon^{4}}+\frac{1}{2 \epsilon^{3}}+\frac{1}{\epsilon^{2}}\left(\frac{51}{24}+\zeta_{2}\right)+\frac{1}{\epsilon}\left(\frac{43}{8}-2 \zeta_{2}+6 \zeta_{3}\right)\right.\right. \\
\left.+\frac{369}{16}+\frac{61}{4} \zeta_{2}-18 \zeta_{4}-24 \zeta_{2} \log 2-3 \zeta_{3}\right] \\
+C_{F} C_{A}\left[\frac{11}{12 \epsilon^{3}}+\frac{1}{\epsilon^{2}}\left(\frac{25}{9}-\frac{1}{2} \zeta_{2}\right)+\frac{1}{\epsilon}\left(\frac{1957}{216}+\frac{13}{2} \zeta_{2}-\frac{15}{2} \zeta_{3}\right)\right. \\
\left.+\frac{31885}{1296}+\frac{38}{3} \zeta_{2}-13 \zeta_{4}+12 \zeta_{2} \log 2+\frac{13}{3} \zeta_{3}\right]
\end{gathered}
$$




$$
\begin{gathered}
-C_{F} C_{A} \frac{n_{\epsilon}}{12}\left[\frac{1}{2 \epsilon^{3}}+\frac{11}{6 \epsilon^{2}}+\frac{1}{\epsilon}\left(\frac{215}{36}+3 \zeta_{2}\right)+\frac{4559}{216}+11 \zeta_{2}+4 \zeta_{3}\right] \\
+C_{F} n_{f} \frac{1}{6}\left[-\frac{1}{\epsilon^{3}}-\frac{8}{3 \epsilon^{2}}-\frac{1}{\epsilon}\left(\frac{149}{18}+6 \zeta_{2}\right)-\frac{3269}{108}-16 \zeta_{2}-8 \zeta_{3}\right] \\
+C_{F} n_{m} \frac{2}{3}\left[\frac{1}{\eta}\left(\frac{1}{\epsilon^{2}}-\frac{5}{3 \epsilon}+\frac{28}{9}+2 \zeta_{2}\right)-\frac{1}{\epsilon^{3}}+\frac{1}{2 \epsilon^{2}}+\frac{1}{\epsilon}\left(-\frac{55}{24}-3 \zeta_{2}\right)\right. \\
\left.\left.+\frac{1675}{432}-2 \zeta_{2}+\zeta_{3}\right]\right\} \\
+a_{e}^{0} a_{s}^{0}\left\{C_{F}^{2} \frac{n_{\epsilon}}{4}\left[-\frac{1}{\epsilon^{3}}-\frac{9}{2 \epsilon^{2}}-\frac{15}{2 \epsilon}-23 \zeta_{2}-2 \zeta_{3}+1\right]\right. \\
\left.+C_{A} C_{F} \frac{n_{\epsilon}}{8}\left[-\frac{11}{\epsilon}-\frac{105}{2}+4 \zeta_{2}+20 \zeta_{3}\right]\right\} \\
+\left(a_{e}^{0}\right)^{2}\left\{\left(C_{F} n_{\epsilon}\right)^{2} \frac{1}{32}\left[-\frac{3}{\epsilon^{2}}-\frac{5}{\epsilon}-30 \zeta_{2}+\frac{85}{2}\right]\right. \\
+C_{F} n_{f} \frac{n_{\epsilon}}{8}\left[\frac{1}{\epsilon^{2}}+\frac{7}{2 \epsilon}+\frac{21}{4}+6 \zeta_{2}\right] \\
+C_{F} n_{\epsilon}\left(\frac{C_{F}}{2}+\frac{n_{\epsilon} C_{A}}{8}-\frac{C_{A}}{4}\right)\left[\frac{1}{\epsilon^{2}}+\frac{2}{\epsilon}-3+4 \zeta_{2}\right] \\
\left.+C_{F} n_{\epsilon} n_{m} \frac{1}{8}\left[\frac{1}{\epsilon^{2}}+\frac{7}{2 \epsilon}-\frac{3}{4}+2 \zeta_{2}\right]\right\}+\mathcal{O}\left(a_{i}^{3}, \epsilon, \eta\right) .
\end{gathered}
$$

The expression for $Z_{q}$ is also included in the supplementary material of the paper. The couplings in (3.12) get renormalized in the $\overline{\mathrm{MS}}$ scheme with $n_{f}+n_{m}$ flavours. We have confirmed that (3.7) holds after the heavy $n_{h}$ flavours are decoupled in $F_{1}(M, m, s)[27,56]$.

The anomalous $1 / \eta$ terms are cancelled between $Z_{q}$ and $\mathcal{S}$. However, because $Z_{q}$ contains $s^{-\eta}$, while $\mathcal{S}$ contains $(m M)^{\eta}$, this gives rise to new, factorization-breaking logarithms of the form $\log (m M / s)$. This is related to the breaking of a scaling symmetry between collinear and soft modes in SCET [50, 51].

Finally, there is the ultrasoft region defined through $(2.7 \mathrm{~d})$. This region contributes to individual master integrals and even diagrams. As expected, at leading order in $\lambda$, the ultrasoft contributions to the form factors cancel. Hence, in (3.7) no such contribution is present.

The factorization (3.7) also holds for $F_{2}$ and $F_{3}$. However, these cases are rather trivial at NNLO. Since $F_{2}$ and $F_{3}$ vanish at leading order and the soft contribution only enters at NNLO, there are only hard and collinear contributions that are non-vanishing. Furthermore, only the NLO term of $Z_{q}$ is required and the factorization anomaly does not enter at this order.

\section{Comparison with heavy-quark form factor}

Small mass effects have been considered before in the literature. In this section we compare our findings to previous results and consider observables other than the heavy-to-light form factor. 
To start with, the collinear contribution, $Z_{q}$, given in (3.12) agrees with a corresponding expression obtained in [5] apart from the $n_{m}$ terms that were not considered there. These terms that lead to the factorization anomaly have been considered in [6], in particular in the context of the heavy-quark form factor $F_{1}(m, m, s)$. In [6] the soft contribution to the heavy-quark form factor, $\mathcal{S}^{\prime}$, was defined as

$$
\mathcal{S}_{[6]}^{\prime}=1+\left(\frac{\alpha_{s}}{4 \pi}\right)^{2} C_{F} \int[\mathrm{d} k] \frac{\left(-2 p^{\mu}\right)\left(-2 q^{\nu}\right)}{\left(k^{2}\right)^{2}(2 p \cdot k)(2 q \cdot k)} \Pi_{\mu \nu}^{\left(n_{m}\right)}(k) .
$$

This definition is motivated by the eikonal approximation and does not lead to a factorization anomaly. Our definition of the soft contribution to the heavy-quark form factor is motivated by SCET. For consistency with the collinear contribution, one also has to introduce the same regulator here. Our definition therefore reads

$$
\mathcal{S}^{\prime}=1+\left(\frac{\alpha_{s}}{4 \pi}\right)^{2} C_{F} \int[\mathrm{d} k] \frac{\left(-2 p_{+}^{\mu}\right)\left(-2 q_{-}^{\nu}\right)}{\left(k^{2}\right)^{2}\left(2 p_{+} \cdot k\right)^{1+\eta}\left(2 q_{-} \cdot k\right)} \Pi_{\mu \nu}^{\left(n_{m}\right)}(k),
$$

where $p$ is assumed to scale anti-collinear and $q$ collinear. Because any integral of the form

$$
I\left(n_{1}, n_{2}, n_{3}\right) \equiv \int[\mathrm{d} k] \frac{1}{\left(k^{2}\right)^{n_{1}}\left(2 p_{+} \cdot k\right)^{n_{2}}\left(2 q_{-} \cdot k\right)^{n_{3}}} \Pi_{\mu \nu}^{\left(n_{m}\right)}(k)
$$

depends on $p_{+}$and $q_{-}$only through $s=2 p_{+} \cdot q_{-}$, it is invariant under simultaneous rescaling $p_{+} \rightarrow \lambda p_{+}, q_{-} \rightarrow q_{-} / \lambda$. This implies $I\left(n_{1}, n_{2}, n_{3}\right)=\lambda^{-n_{2}} \lambda^{n_{3}} I\left(n_{1}, n_{2}, n_{3}\right)$ and, hence, $I=0$ unless $n_{2}=n_{3}$. However, due to the regulator (3.9), $n_{2}$ can never be equal to $n_{3}$. Hence, all occurring integrals vanish and $\mathcal{S}^{\prime}=1$ at two loops. For the heavy-quark form factor, the factorization anomaly in $Z_{q}$ is therefore not cancelled by an anomaly in the soft contribution. In the following we show that, instead, it is cancelled by an anomaly in $\bar{Z}_{q}$. This is a contribution analogous to $Z_{q}$, but due to the anti-collinear fermion.

In section 3.3 we treat the factorization anomaly by shifting the propagators of the heavy fermion. Previously, only the expansion in the collinear direction $e$ contributed because $p^{2}$ was large. Now, however, we also have to expand in the anti-collinear direction $\bar{e}$, which results in a new term, $\bar{Z}_{q}$. All non-anomalous terms in $\bar{Z}_{q}$ are identical to $Z_{q}$ because $e$ and $\bar{e}$ can be interchanged while simultaneously swapping $p$ and $q$. This is, however, not valid for the anomalous $n_{m}$ terms. In order for the regularization scheme to be consistent, the propagator that is regularized must remain the same throughout the calculation. Thereby, the $e \leftrightarrow \bar{e}$ symmetry is broken for the $n_{m}$ terms [55]. As a consequence, the $n_{m}$ terms of $\bar{Z}_{q}$ have to be obtained through an explicit calculation. We find

$$
\begin{aligned}
\left.\sqrt{\bar{Z}_{q}}\right|_{n_{m}}=\left(a_{s}^{0}\left(m^{2}\right)\right)^{2} C_{F} n_{m} \frac{2}{3}[- & \frac{1}{\eta}\left(\frac{1}{\epsilon^{2}}-\frac{5}{3 \epsilon}+\frac{28}{9}+2 \zeta_{2}\right)+\frac{1}{2 \epsilon^{3}}-\frac{5}{6 \epsilon^{2}}-\frac{253}{72 \epsilon} \\
& \left.+\frac{5083}{432}-\frac{14}{3} \zeta_{2}-\zeta_{3}\right]+\mathcal{O}\left(a_{s}^{3}, \epsilon, \eta\right) .
\end{aligned}
$$

This way the relevant expression $Z_{q} \times \bar{Z}_{q}$ is anomaly free. We can test this result by checking the relation

$$
F_{1}(m, m, s)=\sqrt{Z_{q} \times \bar{Z}_{q}} \times \mathcal{S}^{\prime} \times F_{1}(0,0, s)+\mathcal{O}\left(m^{2} / s\right) .
$$


The CDR result of the heavy-quark form factor $F_{1}(m, m, s)$ can, for example, be obtained from [57], while the massless case $F_{1}(0,0, s)$ can be found in [58]. Further, we have checked explicitly that (4.5) also holds in $n_{\epsilon} \mathrm{QCD}$ by using the FDH results from [27].

Because of these differences in the calculation of $\mathcal{S}^{\prime}$ and $\bar{Z}_{q}$, we do not expect agreement beyond the pure QCD contributions with $[5,6]$. However, in accordance with the previous results, we find

$$
Z_{[5]}=\left.\sqrt{Z_{q} \times \bar{Z}_{q}}\right|_{n_{m}, n_{\epsilon} \rightarrow 0}
$$

and

$$
Z_{[6]} \times \mathcal{S}_{[6]}^{\prime}=\sqrt{Z_{q} \times \bar{Z}_{q}} \times\left.\mathcal{S}^{\prime}\right|_{n_{\epsilon} \rightarrow 0} .
$$

Hence, our results agree with previous ones but extend them to processes where additional fermions with a large mass are present. This agreement as well as the fact that $Z_{q}$ is the same for $F_{1}(M, m, s)$ and $F_{1}(m, m, s)$ is a strong indication that the factorization presented here is general. Thus, it is possible to obtain the leading small-mass terms of scattering amplitudes such as muon-electron scattering as follows: the hard part can be obtained from the corresponding amplitude with $m=0$. For each external fermion of mass $m$, we multiply by the corresponding $\sqrt{Z_{q}}$ or $\sqrt{\bar{Z}_{q}}$. In addition, we have to compute the process-dependent soft contribution according to the prescription exemplified in (3.8) and (4.2).

\section{Conclusions}

The purpose of this article is to facilitate the future computation of amplitudes at NNLO for processes with two different non-vanishing fermion masses. In particular, we are interested in the relation of such amplitudes for the case where the smaller mass $m$ is kept, compared to the case where it is set to zero. Since fermion masses in the Standard Model have a rather strong hierarchy, the full dependence on $m$ is often not required. This is particularly the case for QED processes with muons and electrons.

As a starting point, we have calculated the heavy-to-light form factors at NNLO in QCD (and QED). We have done this by keeping the full mass dependence as well as by expanding in $m$ by employing the method of regions. We have verified that the leading term of the expanded result approximates the full result very well. For QED we have also computed the terms of order $m$, resulting in an even better approximation.

At leading order in this expansion the relation between the massless and massive results can be written in a factorized form. The presence of a second large mass leads to a factorization (or collinear) anomaly in the soft contribution. Hence, our scheme for the soft part differs from previous expressions. We have also independently calculated the mass factorization functions $Z_{q}$ and $\bar{Z}_{q}$ from the heavy-to-light form factor and the heavy-quark form factor, respectively. This has been done by identifying the collinear contribution to the form factors at the diagrammatic level and evaluating them. Our expressions for $Z_{q}$ and $\bar{Z}_{q}$ agree with previously obtained results $[5,6]$ for the purely gluonic 
terms, where no anomaly is present. Combining our expressions of soft and (anti-)collinear contributions, the factorization anomaly cancels in all cases. We have also reproduced previously discussed factorized expressions for leading small-mass terms in the case where only one fermion mass is present. Taken together, this provides strong evidence that our factorization is universally valid for processes with two different non-vanishing masses with a strong hierarchy.

A particular application we have in mind is to obtain the leading electron mass terms of the NNLO amplitude for muon-electron scattering. This is relevant for the proposed MUonE experiment. The additional ingredients required are the NNLO amplitude for massless electrons, that corresponds to the hard part, as well as the process-specific soft contribution. We have provided a consistent definition of the latter contribution and found that its computation is much simpler than the calculation of the hard part.

\section{Acknowledgments}

We are grateful to Sophia Borowka, Seraina Glaus, and Michael Spira for fruitful discussions on numerical evaluation of loop integrals. We want to further thank Andrea Visconti for sharing his notes on the FDH calculation of $F_{1}(M, 0, s)$. TE and YU are supported by the Swiss National Science Foundation (SNF) under contract 200021_178967 and 200021_163466, respectively.

\section{A Master integrals}

The determination of the form factors (2.1) requires the calculation of 40 scalar two-loop master integrals that are presented in this appendix. The integrals are graphically defined in figure 4 and normalized to

$$
[\mathrm{d} k]=\left(\frac{\mu^{2}}{M^{2}}\right)^{\epsilon} \Gamma(1-\epsilon) \frac{\mathrm{d}^{d} k}{i \pi^{d / 2}} .
$$

One integral, 6TopP1, is not fully defined this way because it contains a numerator. It is explicitly given by

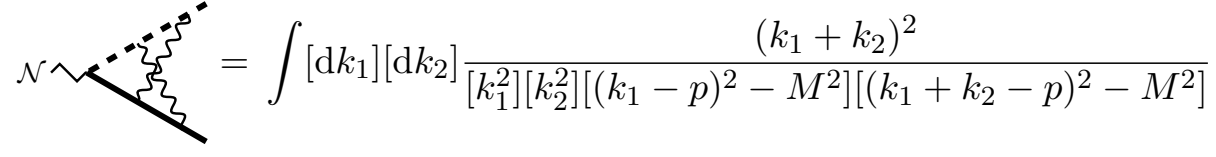

$$
\begin{aligned}
& \times \frac{1}{\left[\left(k_{2}-q\right)^{2}-m^{2}\right]\left[\left(k_{1}+k_{2}-q\right)^{2}-m^{2}\right]} .
\end{aligned}
$$

We calculated these integrals using the method of regions [37]. Motivated by SCET [4749], we expect hard, soft, and collinear regions to appear. In fact, individual integrals can contain ultrasoft regions that drop out of the final result at leading order in $z$. The existence of such regions has been noted before [59] as has the fact that they drop out of the final result [6]. 


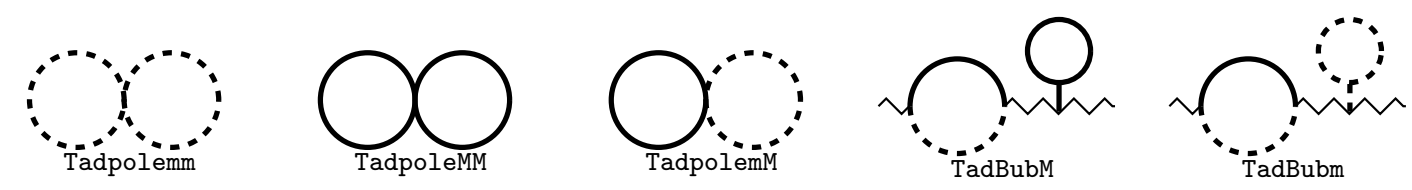

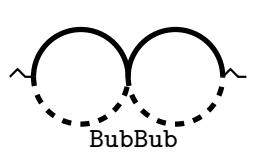
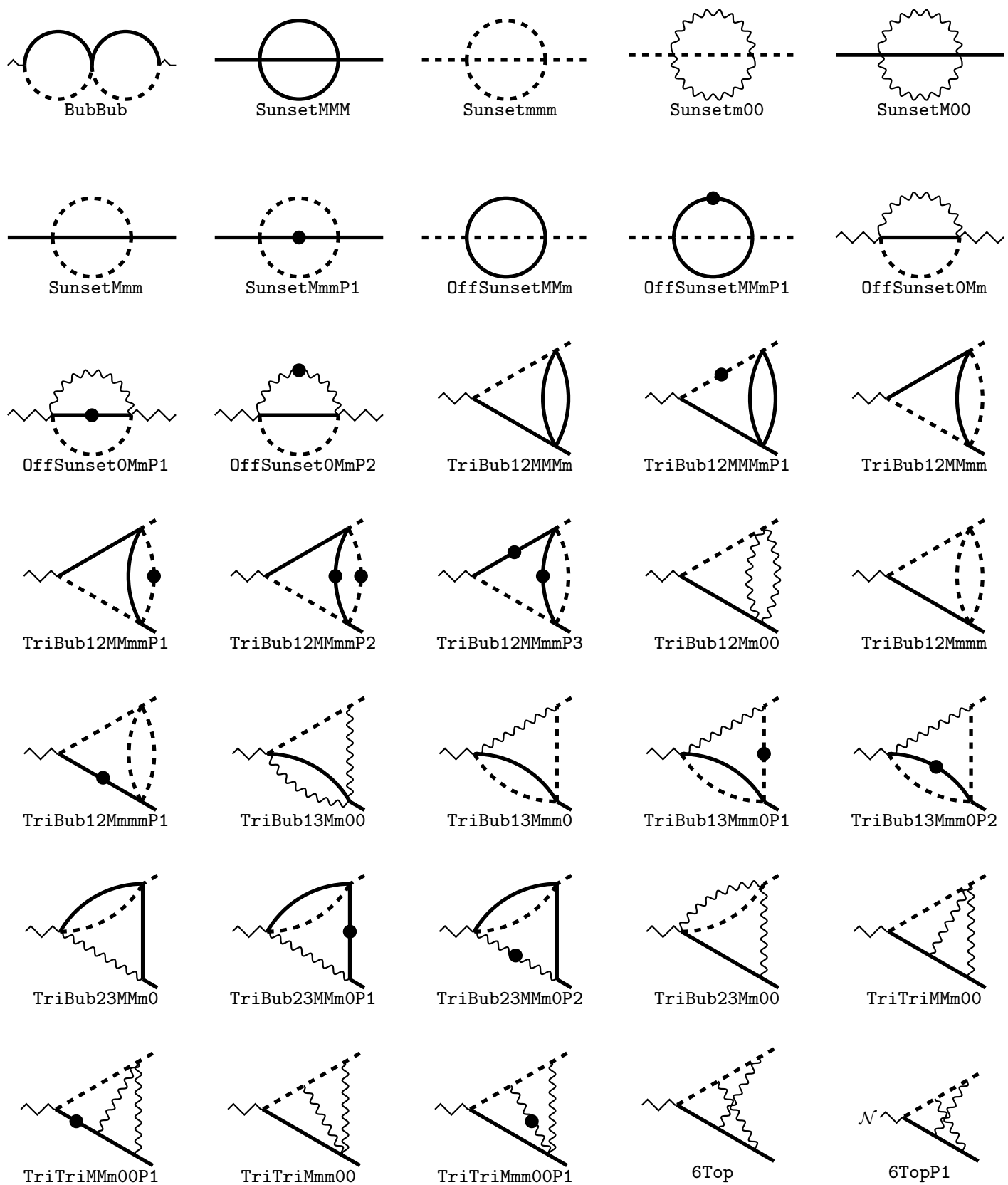

Figure 4. The 40 scalar two-loop master integrals that contribute to the heavy-to-light form factors. For the definition of the numerator of 6TopP1, see (A.2). The solid and dashed thick lines correspond to the heavy and light fermions, respectively. The wavy and zigzag lines correspond to massless gauge bosons and the external current. The heavy (light) fermion momentum $p(q)$ is taken to be incoming (outgoing). A dot indicates that the corresponding propagator is squared. 
There are two equivalent formulations of the method of regions: expanding the momentum representation of the integral in momentum regions using light-cone coordinates or expanding in the alpha representation after Feynman parametrization [59]. In the former, the $k_{+}, k_{-}$, and $k_{\perp}$ components of the loop momentum are scaled differently and then expanded. This way one obtains the regions defined in (2.7). In the alpha representation one instead scales the Feynman parameters. The revelation of regions in this representation has been automatized in the Mathematica program asy [60, 61] based on geometric properties of scaleless integrals. We used both methods concurrently.

The leading hard contribution of integrals always corresponds to the matching massless integral [26, 62]. Subleading terms can also be matched using a Passarino-Veltman decomposition and integral reduction.

Collinear, soft, and ultrasoft contributions can be obtained using Mellin-Barnes decomposition. In our case, the techniques developed in [63, 64] were sufficient to calculate all integrals. For more complicated (multiple) Mellin-Barnes integrals, we used the programs MB.m [65], MBresolve.m [66], and barnesroutines.m [67] to resolve and simplify the integrals and, in rare cases, sum residues with XSummer $[68,69]$. When encountering hypergeometric functions, we expanded them using HypExp [70] to harmonic polylogarithms $[45,46]$.

All expanded integral solutions were numerically compared with the full integrals [28] as well as the numerical program FIESTA [71] for various values of $z$.

The analytic expression expanded in $m$ for all 40 integrals can be found in the supplementary material.

Open Access. This article is distributed under the terms of the Creative Commons Attribution License (CC-BY 4.0), which permits any use, distribution and reproduction in any medium, provided the original author(s) and source are credited.

\section{References}

[1] T. Becher and M. Neubert, On the structure of infrared singularities of gauge-theory amplitudes, JHEP 06 (2009) 081 [Erratum ibid. 11 (2013) 024] [arXiv:0903.1126] [INSPIRE].

[2] E. Gardi and L. Magnea, Factorization constraints for soft anomalous dimensions in QCD scattering amplitudes, JHEP 03 (2009) 079 [arXiv:0901.1091] [INSPIRE].

[3] T. Becher and M. Neubert, Infrared singularities of QCD amplitudes with massive partons, Phys. Rev. D 79 (2009) 125004 [Erratum ibid. D 80 (2009) 109901] [arXiv:0904.1021] [INSPIRE].

[4] A.A. Penin, Two-loop photonic corrections to massive Bhabha scattering, Nucl. Phys. B $\mathbf{7 3 4}$ (2006) 185 [hep-ph/0508127] [INSPIRE].

[5] A. Mitov and S. Moch, The singular behavior of massive QCD amplitudes, JHEP 05 (2007) 001 [hep-ph/0612149] [INSPIRE].

[6] T. Becher and K. Melnikov, Two-loop QED corrections to Bhabha scattering, JHEP 06 (2007) 084 [arXiv:0704.3582] [INSPIRE]. 
[7] T. Liu, A.A. Penin and N. Zerf, Three-loop quark form factor at high energy: the leading mass corrections, Phys. Lett. B 771 (2017) 492 [arXiv:1705.07910] [INSPIRE].

[8] T. Liu and A. Penin, High-energy limit of mass-suppressed amplitudes in gauge theories, JHEP 11 (2018) 158 [arXiv: 1809.04950] [INSPIRE].

[9] J. Blümlein, P. Marquard and N. Rana, Asymptotic behavior of the heavy quark form factors at higher order, Phys. Rev. D 99 (2019) 016013 [arXiv:1810.08943] [INSPIRE].

[10] G. Abbiendi et al., Measuring the leading hadronic contribution to the muon $g-2$ via $\mu$ e scattering, Eur. Phys. J. C 77 (2017) 139 [arXiv:1609.08987] [INSPIRE].

[11] MUONE collaboration, Measurement of the leading hadronic contribution to the muon $g-2$ via space-like data, PoS (EPS-HEP2017) 358.

[12] M. Alacevich et al., Muon-electron scattering at NLO, arXiv:1811.06743 [INSPIRE].

[13] M. Fael, Hadronic corrections to $\mu$-e scattering at NNLO with space-like data, JHEP 02 (2019) 027 [arXiv : 1808. 08233] [INSPIRE].

[14] M. Fael and M. Passera, Muon-electron scattering at NNLO: the hadronic corrections, arXiv:1901.03106 [INSPIRE].

[15] P. Mastrolia, M. Passera, A. Primo and U. Schubert, Master integrals for the NNLO virtual corrections to $\mu$ e scattering in QED: the planar graphs, JHEP 11 (2017) 198 [arXiv: 1709.07435] [INSPIRE].

[16] S. Di Vita et al., Master integrals for the NNLO virtual corrections to $\mu$ e scattering in QED: the non-planar graphs, JHEP 09 (2018) 016 [arXiv:1806.08241] [INSPIRE].

[17] T. Kinoshita and A. Sirlin, Radiative corrections to Fermi interactions, Phys. Rev. 113 (1959) 1652 [INSPIRE].

[18] R.E. Behrends, R.J. Finkelstein and A. Sirlin, Radiative corrections to decay processes, Phys. Rev. 101 (1956) 866 [INSPIRE].

[19] T. van Ritbergen and R.G. Stuart, On the precise determination of the Fermi coupling constant from the muon lifetime, Nucl. Phys. B 564 (2000) 343 [hep-ph/9904240] [INSPIRE].

[20] A. Arbuzov, A. Czarnecki and A. Gaponenko, Muon decay spectrum: leading logarithmic approximation, Phys. Rev. D 65 (2002) 113006 [hep-ph/0202102] [INSPIRE].

[21] A. Arbuzov and K. Melnikov, $O\left(\alpha^{2} \ln \left(m_{\mu} / m_{e}\right)\right)$ corrections to electron energy spectrum in muon decay, Phys. Rev. D 66 (2002) 093003 [hep-ph/0205172] [INSPIRE].

[22] C. Anastasiou, K. Melnikov and F. Petriello, The electron energy spectrum in muon decay through O(alpha**2), JHEP 09 (2007) 014 [hep-ph/0505069] [INSPIRE].

[23] R. Bonciani and A. Ferroglia, Two-loop QCD corrections to the heavy-to-light quark decay, JHEP 11 (2008) 065 [arXiv: 0809.4687] [INSPIRE].

[24] H.M. Asatrian, C. Greub and B.D. Pecjak, NNLO corrections to $\bar{B} \rightarrow X_{u} l \bar{\nu}$ in the shape-function region, Phys. Rev. D 78 (2008) 114028 [arXiv:0810.0987] [INSPIRE].

[25] M. Beneke, T. Huber and X.Q. Li, Two-loop QCD correction to differential semi-leptonic $b \rightarrow u$ decays in the shape-function region, Nucl. Phys. B 811 (2009) 77 [arXiv:0810.1230] [INSPIRE].

[26] G. Bell, Higher order QCD corrections in exclusive charmless $B$ decays, Ph.D. thesis, Munich University, Munich, Germany (2006), arXiv:0705.3133 [INSPIRE]. 
[27] C. Gnendiger, A. Signer and A. Visconti, Regularization-scheme dependence of QCD amplitudes in the massive case, JHEP 10 (2016) 034 [arXiv:1607.08241] [INSPIRE].

[28] L.-B. Chen, Two-Loop master integrals for heavy-to-light form factors of two different massive fermions, JHEP 02 (2018) 066 [arXiv:1801.01033] [INSPIRE].

[29] A.B. Goncharov, Multiple polylogarithms, cyclotomy and modular complexes, Math. Res. Lett. 5 (1998) 497 [arXiv:1105.2076] [InSPIRE].

[30] S. Berman and A. Sirlin, Some considerations on the radiative corrections to muon and neutron decay, Ann. Phys. 20 (1962) 20.

[31] C. Gnendiger, A. Signer and D. Stöckinger, The infrared structure of QCD amplitudes and $H \rightarrow g g$ in FDH and DRED, Phys. Lett. B 733 (2014) 296 [arXiv:1404.2171] [INSPIRE].

[32] D.J. Broadhurst, N. Gray and K. Schilcher, Gauge invariant on-shell $Z_{2}$ in $Q E D, Q C D$ and the effective field theory of a static quark, Z. Phys. C 52 (1991) 111 [INSPIRE].

[33] A. von Manteuffel and C. Studerus, Reduze 2 - Distributed Feynman Integral Reduction, arXiv: 1201.4330 [INSPIRE].

[34] S. Laporta, High precision calculation of multiloop Feynman integrals by difference equations, Int. J. Mod. Phys. A 15 (2000) 5087 [hep-ph/0102033] [inSPIRE].

[35] J. Vollinga and S. Weinzierl, Numerical evaluation of multiple polylogarithms, Comput. Phys. Commun. 167 (2005) 177 [hep-ph/0410259] [INSPIRE].

[36] H. Frellesvig, D. Tommasini and C. Wever, On the reduction of generalized polylogarithms to $L i_{n}$ and $L i_{2,2}$ and on the evaluation thereof, JHEP 03 (2016) 189 [arXiv:1601.02649] [INSPIRE].

[37] M. Beneke and V.A. Smirnov, Asymptotic expansion of Feynman integrals near threshold, Nucl. Phys. B 522 (1998) 321 [hep-ph/9711391] [INSPIRE].

[38] C.G. Bollini and J.J. Giambiagi, Dimensional renormalization: the number of dimensions as a regularizing parameter, Nuovo Cim. B 12 (1972) 20 [INSPIRE].

[39] G. 't Hooft and M.J.G. Veltman, Regularization and renormalization of gauge fields, Nucl. Phys. B 44 (1972) 189 [INSPIRE].

[40] J. C. Collins, Renormalization, An introduction to renormalization, the renormalization group, and the operator-product expansion, Cambridge University Press, Cambridge U.K. (1984).

[41] Z. Bern and D.A. Kosower, The computation of loop amplitudes in gauge theories, Nucl. Phys. B 379 (1992) 451 [INSPIRE].

[42] W. Siegel, Supersymmetric dimensional regularization via dimensional reduction, Phys. Lett. 84B (1979) 193 [INSPIRE].

[43] D.M. Capper, D.R.T. Jones and P. van Nieuwenhuizen, Regularization by dimensional reduction of supersymmetric and nonsupersymmetric gauge theories, Nucl. Phys. B 167 (1980) 479 [INSPIRE].

[44] C. Gnendiger et al., To d, or not to d: recent developments and comparisons of regularization schemes, Eur. Phys. J. C 77 (2017) 471 [arXiv:1705.01827] [INSPIRE].

[45] E. Remiddi and J.A.M. Vermaseren, Harmonic polylogarithms, Int. J. Mod. Phys. A 15 (2000) 725 [hep-ph/9905237] [INSPIRE]. 
[46] D. Maître, HPL, a Mathematica implementation of the harmonic polylogarithms, Comput. Phys. Commun. 174 (2006) 222 [hep-ph/0507152] [INSPIRE].

[47] C.W. Bauer, D. Pirjol and I.W. Stewart, Soft collinear factorization in effective field theory, Phys. Rev. D 65 (2002) 054022 [hep-ph/0109045] [inSPIRE].

[48] C.W. Bauer, S. Fleming, D. Pirjol and I.W. Stewart, An effective field theory for collinear and soft gluons: heavy to light decays, Phys. Rev. D 63 (2001) 114020 [hep-ph/0011336] [INSPIRE].

[49] M. Beneke, A.P. Chapovsky, M. Diehl and T. Feldmann, Soft collinear effective theory and heavy to light currents beyond leading power, Nucl. Phys. B 643 (2002) 431 [hep-ph/0206152] [INSPIRE].

[50] T. Becher and M. Neubert, Drell-Yan production at small $q_{T}$, transverse parton distributions and the collinear anomaly, Eur. Phys. J. C 71 (2011) 1665 [arXiv:1007.4005] [INSPIRE].

[51] T. Becher, G. Bell and M. Neubert, Factorization and resummation for jet broadening, Phys. Lett. B 704 (2011) 276 [arXiv:1104.4108] [INSPIRE].

[52] M. Beneke, Soft-collinear effective theory, in the proceedings of the Helmholtz International Summer School: Heavy Quark Physics, June 6-16, Dubna, Russia (2005).

[53] J.-y. Chiu, A. Jain, D. Neill and I.Z. Rothstein, The rapidity renormalization group, Phys. Rev. Lett. 108 (2012) 151601 [arXiv:1104.0881] [INSPIRE].

[54] V.A. Smirnov, Asymptotic expansions of two loop Feynman diagrams in the Sudakov limit, Phys. Lett. B 404 (1997) 101 [hep-ph/9703357] [INSPIRE].

[55] T. Becher, A. Broggio and A. Ferroglia, Introduction to soft-collinear effective theory, Lect. Notes Phys. 896 (2015) 1 [arXiv:1410.1892].

[56] K.G. Chetyrkin, B.A. Kniehl and M. Steinhauser, Decoupling relations to $O\left(\alpha_{S}^{3}\right)$ and their connection to low-energy theorems, Nucl. Phys. B 510 (1998) 61 [hep-ph/9708255] [INSPIRE].

[57] W. Bernreuther et al., Two-loop QCD corrections to the heavy quark form-factors: The Vector contributions, Nucl. Phys. B 706 (2005) 245 [hep-ph/0406046] [INSPIRE].

[58] T. Gehrmann, T. Huber and D. Maître, Two-loop quark and gluon form-factors in dimensional regularisation, Phys. Lett. B 622 (2005) 295 [hep-ph/0507061] [INSPIRE].

[59] V.A. Smirnov, Problems of the strategy of regions, Phys. Lett. B 465 (1999) 226 [hep-ph/9907471] [inSPIRE].

[60] A. Pak and A. Smirnov, Geometric approach to asymptotic expansion of Feynman integrals, Eur. Phys. J. C 71 (2011) 1626 [arXiv:1011.4863] [INSPIRE].

[61] B. Jantzen, A.V. Smirnov and V.A. Smirnov, Expansion by regions: revealing potential and Glauber regions automatically, Eur. Phys. J. C 72 (2012) 2139 [arXiv:1206. 0546] [INSPIRE].

[62] A. Ferroglia, C. Greub, A. Sirlin and Z. Zhang, Contributions of the W-boson propagator to $\mu$ and $\tau$ leptonic decay rates, Phys. Rev. D 88 (2013) 033012 [arXiv:1307.6900] [INSPIRE].

[63] V.A. Smirnov, Analytical result for dimensionally regularized massless on shell double box, Phys. Lett. B 460 (1999) 397 [hep-ph/9905323] [INSPIRE].

[64] J.B. Tausk, Nonplanar massless two loop Feynman diagrams with four on-shell legs, Phys. Lett. B 469 (1999) 225 [hep-ph/9909506] [INSPIRE]. 
[65] M. Czakon, Automatized analytic continuation of Mellin-Barnes integrals, Comput. Phys. Commun. 175 (2006) 559 [hep-ph/0511200] [INSPIRE].

[66] A.V. Smirnov and V.A. Smirnov, On the resolution of singularities of multiple Mellin-Barnes integrals, Eur. Phys. J. C 62 (2009) 445 [arXiv:0901.0386] [INSPIRE].

[67] D. Kosower, barnesroutines.m, https://mbtools.hepforge.org (2007).

[68] S. Moch, P. Uwer and S. Weinzierl, Nested sums, expansion of transcendental functions and multiscale multiloop integrals, J. Math. Phys. 43 (2002) 3363 [hep-ph/0110083] [InSPIRE].

[69] S. Moch and P. Uwer, XSummer: transcendental functions and symbolic summation in form, Comput. Phys. Commun. 174 (2006) 759 [math-ph/0508008] [INSPIRE].

[70] T. Huber and D. Maître, HypExp: a Mathematica package for expanding hypergeometric functions around integer-valued parameters, Comput. Phys. Commun. 175 (2006) 122 [hep-ph/0507094] [INSPIRE].

[71] A.V. Smirnov, FIESTA4: optimized Feynman integral calculations with GPU support, Comput. Phys. Commun. 204 (2016) 189 [arXiv:1511.03614] [INSPIRE]. 